\title{
Elementary Particle Approach to Some Nuclear Pion Reactions.
}

\author{
P. Pascual \\ CERN - Geneva \\ A. FuJII \\ Institut de Physique Nucléaire - Orsay \\ (Nuovo Cimento. $65 \mathrm{~A}, 411(1970))$
}

In the paper of ref. $\left({ }^{3}\right)$ a wrong value of $/ \tau_{\frac{1}{2}}$ for ${ }^{6}$ He was given. Due to this fact the results obtained in Sect. 7 of the present paper must be modified as follows:

... For the radiative pion capture we have $t-\ldots-1.7882 \cdot 10^{+4}$ (MeV) and we obtain

$$
F^{(0.1)}(l):=-(1.463+0.0996) \cdot 10^{+4} \mathrm{Me} \mathrm{V}^{\mathrm{T}}
$$

'Then we obtain

$$
\Gamma\left(\pi^{-} \rightarrow \gamma\right)=(1.86 \therefore 0.18) \cdot 10^{+15} \mathrm{~s} .
$$

There is not a direct measurement of this quantity. Nevertheless two important quantities have been measured: the pion disappearence width from the ls orbit

$$
\Gamma_{1 s}=(0.15 \pm 0.05) \mathrm{keV}\left({ }^{11}\right), \quad \Gamma_{1 s}=(0.39 \pm 0.36) \mathrm{ke}^{\circ}\left({ }^{12}\right)
$$

and the branching ratio to the radiative pion capture channel $\left({ }^{13}\right)$

$$
R \equiv \frac{\Gamma\left[\left(\pi^{-}+{ }^{6} \mathrm{I} i\right)_{1 s, 2 s, 2} \ldots \rightarrow{ }^{6} \mathrm{He}+\gamma\right]}{\Gamma\left[\left(\pi^{-}+{ }^{6} \mathrm{~L}, \mathrm{i}\right)_{1 s, 2 s, 2) \ldots} \rightarrow \mathrm{all}\right]}=0.010 \pm 0.001
$$

If the pion-capture rate takes place tolally from the $1 s$ orbit we obtain from (32) and (33) the values

$$
\Gamma\left[\left(\pi^{-}+{ }^{6} \mathrm{Li}\right)_{1 s} \rightarrow{ }^{6} \mathrm{He}+\gamma\right]=(2.3 \dashv 0.8) \cdot 10^{+15} \mathrm{~s}^{-1}\left({ }^{11}\right)
$$

and $(5.9 \pm 5.5) \cdot 10^{-15} \mathrm{~s}^{-1}\left({ }^{13}\right)$ which must be comparable with the value given in eq. (31). As we see the agreement is very good. 